Accepted for Ap.J. Vol. 502, July 20, 1998

\title{
Astrometry and Photometry for Two Dwarf Carbon Stars
}

\author{
Hugh C. Harris, Conard C. Dahn, Richard L. Walker, Christian B. Luginbuhl, \\ Alice K.B. Monet, Harry H. Guetter, Ronald C. Stone, Fredrick J. Vrba, \\ David G. Monet, and Jeffrey R. Pier \\ U.S. Naval Observatory, Flagstaff, AZ 86002
}

\begin{abstract}
Preliminary trigonometric parallaxes and BVI photometry are presented for two dwarf carbon stars, LP765-18 (= LHS1075) and LP328-57 (= CLS96). The data are combined with the literature values for a third dwarf carbon star, G77-61 (= LHS1555). All three stars have very similar luminosities $\left(9.6<\mathrm{M}_{\mathrm{V}}<10.0\right)$ and very similar broadband colors across the entire visual-to-near IR (BVIJHK) wavelength range. Their visual (BVI) colors differ from all known red dwarfs, subdwarfs, and white dwarfs. In the $\mathrm{M}_{\mathrm{V}}$ versus $\mathrm{V}-\mathrm{I}$ color-magnitude diagram they are approximately 2 magnitudes subluminous compared with normal disk dwarfs with solar-like metallicities, occupying a region also populated by O-rich subdwarfs with $-1.5<[\mathrm{m} / \mathrm{H}]<-1.0$. The kinematics indicate that they are members of the Galactic spheroid population. The subluminosity of all three stars is due to an as-yet-unknown combination of (undoubtedly low) metallicity, possibly enhanced helium abundance, and unusual line-blanketing in the bandpasses considered. The properties of the stars are compared with models for the production of dwarf carbon stars.
\end{abstract}

Subject headings: astrometry — stars: carbon — stars: population II

\section{Introduction}

The discovery of the first dwarf carbon star (Dahn et al. 1977) sparked considerable research on the evolution of stars to and through the dwarf carbon star $(\mathrm{dC})$ stage. It is now accepted that $\mathrm{dC}$ stars are formed through the accretion of carbon-rich material from a close companion star that was evolving on the asymptotic giant branch at the time of mass transfer. They are now recognized as members of a larger family of stars that have undergone mass-transfer binary evolution, a family that includes the halo $\mathrm{CH}$ giant stars, the disk Ba giants, the Ba dwarf or $\mathrm{CH}$ subgiant stars, and the extrinsic S stars (Green 1997).

De Kool \& Green (1995) have modeled dC star formation by following the evolution of a large variety of binary systems constructed from the observed distributions of component masses, 
orbital separations, and metallicities for unevolved binaries. Although these simulations involve a large number of poorly constrained parameters, the results indicate that the formation of a $\mathrm{dC}$ star is strongly favored by low initial metallicity, and that virtually no dCs are produced in systems with metallicities above half solar. Furthermore, the mass distribution is found to peak strongly in the 0.4 to 0.9 solar mass range.

Despite the studies to date, our understanding of $\mathrm{dC}$ stars remains highly speculative, based as much on plausibility arguments as on objective facts. Distances of these stars are among the most essential missing data, and must be known in order to determine each star's luminosity, space velocity, mass, and evolutionary status. Presently, only one dC star, G77-61, has a reliable trigonometric distance determination and it indicates a high space velocity and - by implication low metallicity. This implied low metallicity is consistent with the detailed atmospheric analysis of G77-61 by Gass et al. (1988) who derived the extremely low metallicity of $[\mathrm{Fe} / \mathrm{H}]=-5.6$, a value which certainly implies very early epoch halo formation.

A larger sample of $\mathrm{dC}$ stars with properties well constrained by fundamental observational data is clearly needed to understand these stars as a class. Unfortunately, no known dC stars are bright enough to have had their parallaxes measured with high accuracy by the Hipparcos satellite. In this paper we report preliminary trigonometric parallaxes for two additional dC stars and discuss some of their properties.

\section{Data}

The stars LP765-18 and LP328-57 have been observed since 1992 as part of the Naval Observatory CCD parallax program using a Tektronix 2048 CCD camera on the 1.55-m Strand telescope. This camera is producing relative astrometry more accurate than that previously obtained using a TI 800 CCD (Monet et al. 1992), primarily as a result of the improved reference star frames available in the larger field of view. Experience to date demonstrates that the Tek 2048 program generally produces relative astrometric measurements accurate to \pm 3 mas for a single observation and relative parallaxes accurate to \pm 0.5 mas after roughly 100 observations adequately distributed over the parallactic ellipse and spanning at least 3 years (Dahn 1997). This high accuracy is essential in order to determine accurate distances for even the nearer of the known dC stars, almost all of which are more than 100 pc from the sun.

The two dC stars reported here have 68 observations on 56 nights over 4.2 years (LP765-18) and 106 observations on 86 nights over 4.3 years (LP328-57). The parallax results presented in Table 1 are regarded as preliminary in the sense that both fields are still being observed. The BVI photometry reported are USNO data taken with the 1.0-m telescope using procedures described in Monet et al. (1992), and are accurate to \pm 0.02 magnitudes. The JHK photometric data are from Bothun et al. (1991) and Dearborn et al. (1986). For completeness, Table 1 includes corresponding data for G77-61 (the third dC star with a measured trigonometric parallax) taken 
from the literature.

Also given in Table 1 is the correction $(\Delta \pi)$ necessary to convert the directly measured "relative" parallaxes $\left(\pi_{\text {rel }}\right)$ to "absolute" parallaxes $\left(\pi_{a b s}\right)$. In the cases of LP765-18 and LP328-57, we have used BVI photometry of the individual reference stars to estimate the mean parallax of each reference frame. The uncertainties in the derived $\Delta \pi$ values were estimated as the combination of random uncertainties $\left(\approx \sigma \mathrm{n}^{-1 / 2}\right.$, where $\mathrm{n}$ is the number of stars comprising the reference frame) plus an estimate of possible systematic errors arising from photometric and reddening uncertainties in each field. Because the reference stars in these fields typically have distances of $0.7-2 \mathrm{kpc}$, their estimated parallaxes are small. In the case of G77-61, we adopt the improved statistical estimate for $\Delta \pi$ from van Altena et al. (1995). As can be seen in Table 1, the estimated errors in the $\Delta \pi$ values for these stars are sufficiently small that they contribute only a small amount to the final uncertainties in the absolute parallaxes.

\section{Discussion}

Results interpreted from the parallax data are shown in Table 2. In calculating the formal uncertainties in $\mathrm{M}_{\mathrm{V}}$ and $\mathrm{M}_{\mathrm{K}}$ we have adopted the parallax uncertainties given in Table 1, along with $\pm 0.02 \mathrm{mag}$ for the uncertainties in the $\mathrm{V}$ magnitudes and \pm 0.03 mag for the uncertainties in the $\mathrm{K}$ magnitudes. The tabulated radial velocities $\left(\mathrm{V}_{\mathrm{r}}\right)$ are from Bothun et al. (1991) for LP765-18 and LP328-57 and from Dearborn et al. (1986) for G77-61. The space velocity components $(\mathrm{U}, \mathrm{V}, \mathrm{W})$ in Table 1 have been corrected for a solar motion of $(10,15,7) \mathrm{km} \mathrm{s}^{-1}$ to the local standard of rest; $\mathrm{U}$ is in the direction of the galactic center.

Inspection of Tables 1 and 2 reveals that these three dC stars are remarkably similar in terms of (1) overall kinematic properties, (2) infrared (JHK) colors, (3) optical (BVI) colors, and (4) luminosities $\left(\mathrm{M}_{\mathrm{V}}\right.$ and $\left.\mathrm{M}_{\mathrm{K}}\right)$. Regarding the kinematics specifically, the large overall space velocities

and the large negative $\mathrm{V}$ galactic velocity components are indicative of membership in the Galactic spheroid population.

Regarding the colors, Green et al. (1992) have previously discussed the location of dC stars in the $\mathrm{J}-\mathrm{H}$ versus $\mathrm{H}-\mathrm{K}$ diagram and their apparent separation from the giant and subgiant carbon stars, presumably due (in part) to the higher surface gravities for the dC stars. In Figure 1 we show the location of these $\mathrm{dC}$ stars in the $\mathrm{B}-\mathrm{V}$ versus $\mathrm{V}-\mathrm{I}$ diagram. Because all three were originally identified as stars with high proper motion, we include for comparison a selection of other field stars commonly identified in proper motion surveys: later-type degenerates (open circles), disk dwarfs (filled circles), and metal-poor subdwarfs (crosses). The dC stars occupy a unique region in this diagram. The lone exception to a clear-cut separation for the $\mathrm{dC}$ stars is LP701-29, the only known late-type degenerate with strong CaI absorption which blankets the B bandpass (Dahn et al. 1978). Figure 1 suggests that even broadband photometric surveys of faint, high proper motion stars might succeed in isolating additional $\mathrm{dC}$ candidates. Their location to 
the left of the most extreme metal-poor field subdwarfs known at present suggests metallicities below $[\mathrm{m} / \mathrm{H}] \sim-2$. However, quantitative conclusions about metallicity are not possible because the field subdwarfs have O-rich rather than C-rich atmospheres; with the exception of the analysis of G77-61 by Gass et al. (1988), no complete studies based on model atmospheres have been presented for metal-poor, C-rich stars with dwarf-like gravities.

Figure 2 shows the location of these $\mathrm{dC}$ stars in the $\mathrm{M}_{\mathrm{V}}$ versus $\mathrm{V}-\mathrm{I}$ color magnitude diagram. The solid line represents the observed mean disk main sequence as defined by USNO parallax stars (cf. Monet et al. 1992). The dashed lines show the metal-poor main sequences modeled by Baraffe et al. (1997) for metallicities (scaled from solar) of $[\mathrm{m} / \mathrm{H}]=-1.0,-1.5$ and -2.0 . These authors demonstrated that their models successfully reproduce the main sequence of several globular clusters over this range of metallicities. However, these models are for O-rich atmospheres - not the $\mathrm{C} / \mathrm{O}>1$ compositions which are appropriate for the $\mathrm{dC}$ stars. Furthermore, the position of $\mathrm{dC}$ stars in color-magnitude diagrams cannot necessarily be interpreted simply in terms of metallicity because of the possibility of enhanced helium abundance in their atmospheres, produced by the mass transfer event(s) that increased their carbon abundances. The complicated atmospheric situation and the difficulty in uniquely determining both $\log g$ and the He abundance has been discussed by Gass (1988) and Gass et al. (1988). Therefore, until more detailed spectroscopic investigations are undertaken to derive the helium abundance independently, we are left with the implication from the kinematic information that low metallicity and nearly normal helium abundances are most likely.

Figure 3 shows the location of the $\mathrm{dC}$ stars in the schematic $\mathrm{M}_{\mathrm{K}}$ versus $\mathrm{I}-\mathrm{K}$ diagram. Here the solid line represents the "young disk" stars defined by Leggett (1992) while the dashed lines are again the metal-poor main sequences models from Baraffe et al. (1997). In their analysis of G77-61, Gass et al. (1988) pointed out that the J and K fluxes should not depend strongly on the carbon abundance whereas the fluxes in the B,V,I and $\mathrm{H}$ bandpasses will depend strongly on composition. The three dC stars are also significantly subluminous with respect to the disk main sequence in this diagram. However, it is also clear that going to a redder color index still does not allow a quantitative interpretation in terms of metallicity because the location of G77-61 above the $[\mathrm{m} / \mathrm{H}]=-1.0$ curve is clearly at odds with the value of $[\mathrm{Fe} / \mathrm{H}]=-5.6$ derived by Gass et al. (1988).

Figure 4 shows the location of $\mathrm{G} 77-61$ in the $\mathrm{M}_{\mathrm{V}}$ versus $\mathrm{T}_{\text {eff }}$ plane using the value of $\mathrm{T}_{\text {eff }}=4200^{\circ} \mathrm{K}$ derived by Gass et al. (1988). Once again the dashed lines are from Baraffe et al. (1997). Here we find at least qualitative agreement in that this extremely metal-poor object lies below the $[\mathrm{m} / \mathrm{H}]=-2.0$ curve. This emphasizes the need for higher resolution spectrophotometric observations and C-rich model atmosphere analyses for LP765-18 and LP328-57 in order to establish (at least) $\mathrm{T}_{\text {eff }}$ values for them.

The models of Baraffe et al. (1997) indicate that the derived masses are primarily sensitive to the absolute magnitudes (or luminosities), and only weakly dependent on the colors. 
Acknowledging the dangers of interpreting the $\mathrm{dC}$ stars with O-rich models, the masses so estimated are $0.39 \mathrm{M}_{\odot}$ for LP765-18, $0.37 \mathrm{M}_{\odot}$ for LP328-57, and $0.30 \mathrm{M}_{\odot}$ for G77-61. Models for $\mathrm{dC}$ star formation predict that a range of masses from 0.2 to $1.0 \mathrm{M}_{\odot}$ should exist (de Kool \& Green 1995). However, the frequency distribution of these masses depends critically on the adopted Initial Mass Ratio Distribution (IMRD) for the unevolved binary which is quite uncertain. For a flat IMRD (i.e., $\mathrm{dN} \propto \mathrm{dq}$, where $\mathrm{q}$ is the mass ratio), the distribution for spheroid dC stars peaks rather sharply around $0.7 \mathrm{M}_{\odot}$ at a space density of $\mathrm{dN} / \mathrm{d} \log \mathrm{M}=4 \times 10^{-7} \mathrm{pc}^{-3}$, falling to roughly $\mathrm{dN} / \mathrm{d} \log \mathrm{M}=2 \times 10^{-7} \mathrm{pc}^{-3}$ for stars in the mass range inferred above. On the other hand, for uncorrelated component masses, the models predict a broader peak spanning 0.2 to 0.8 $\mathrm{M}_{\odot}$ with a space density of $\mathrm{dN} / \mathrm{d} \log \mathrm{M}=4 \times 10^{-7} \mathrm{pc}^{-3}$ for spheroid stars. As noted by de Kool \& Green (1995), the observed absolute magnitudes seem to support an IMRD with uncorrelated component masses.

However, the apparent similarity of these three $\mathrm{dC}$ stars is undoubtedly influenced to some extent by selection effects. Hotter, more massive $\mathrm{dC}$ stars are predicted by the models to be common, but hotter stars will have weaker carbon bands and may have spectra and broad-band colors that are not as obviously different from stars with oxygen-rich atmospheres as are these three $\mathrm{dC}$ stars. Another factor that makes the model predictions uncertain is the amount of dilution of the carbon-rich material being transferred from the asymptotic-giant-branch star onto its main-sequence companion: a more massive main sequence star $\left(0.5-1.0 \mathrm{M}_{\odot}\right.$, for example), has a less massive convective envelope than the stars studied here (reducing the dilution), but the carbon-rich material may be mixed into the radiative zone as well (Proffitt \& Michaud 1989), thus increasing the dilution and making hotter $\mathrm{dC}$ stars more difficult to identify.

Furthermore, these three dC stars have been chosen for parallax observations based partly on their unusually-high proper motions. This kinematic selection partially accounts for their all being members of the Galactic spheroid. Disk dC stars may be common - the ratio of disk/spheroid $\mathrm{dC}$ stars is a quantity that will help constrain the models of dC-star formation. Parallax data for a sample of $\mathrm{dC}$ stars with smaller proper motions could help to address this issue. Several such dC stars are probably close enough to get significant parallax measurements - however, none are presently being observed in the Naval Observatory parallax program pending time becoming available in the program at their respective right ascensions.

We conclude that the similar properties of these three $\mathrm{dC}$ stars and their membership in the Galactic spheroid may result in part from the way these stars were selected. Accurate distances for a larger sample of $\mathrm{dC}$ stars are needed, and identification of $\mathrm{dC}$ stars without kinematic bias is essential in order to fully understand how and where $\mathrm{dC}$ stars are produced.

This research has made use of the Simbad database, operated at CDS, Strasbourg, France. 


\section{REFERENCES}

Baraffe, I., Chabrier, G., Allard, F., \& Hauschildt, P.H. 1997, A\&A, 327, 1054

Bothun, G., Elias, J.H., MacAlpine, G., Matthews, K., Mould, J.R., Neugebauer, G., \& Reid, I.N. 1991, AJ, 101, 2220

Dahn, C.C. 1997, in Fundamental Stellar Properties: The Interaction between Observation and Theory, Proc. IAU Symp. 189, ed. T.R. Bedding, A.J. Boothe, \& J. Davis (Dordrecht: Kluwer Academic), 19

Dahn, C.C., Liebert, J., Kron, R.G., Spinrad, H., \& Hintzen, P. 1977, ApJ, 216, 757

Dahn, C.C., Hintzen, P.M., Liebert, J.W., Stockman, H.S., \& Spinrad, H. 1978, ApJ, 219, 979

Dearborn, D.S.P., Liebert, J., Aaronson, M., Dahn, C.C., Harrington, R., Mould, J., \& Greenstein, J.L. 1986, ApJ, 300, 314

de Kool, M., \& Green, P.J. 1995, ApJ, 449, 236

Deutsch, E.W. 1994, PASP, 106, 1134

Gass, H. 1988, A\&A, 193, 185

Gass, H., Liebert, J., \& Wehrse, R. 1988, A\&A, 189, 194

Green, P.J. 1997, in The Carbon Star Phenomenon, Proc. IAU Symp. 177, ed. xxx (Dordrecht: Kluwer), in press

Green, P.J., Margon, B., Anderson, S.F., \& MacConnell, D.J. 1992, ApJ, 400, 659

Leggett, S.K. 1992, ApJS, 82, 351

Monet, D.G., Dahn, C.C., Vrba, F.J., Harris, H.C., Pier, J.R., Luginbuhl, C.B., \& Ables, H.D. 1992, AJ, 103, 638

Proffitt, C.R., \& Michaud, G. 1989, ApJ, 345, 998

van Altena, W.F., Lee, J.T., \& Hoffleit, E.D. 1995, The General Catalogue of Trigonometric Stellar Parallaxes, Fourth Edition (L. Davis Press: Schenectady) 
Table 1. Astrometric and Photometric Data.

\begin{tabular}{|c|c|c|c|c|c|c|c|c|c|c|}
\hline $\begin{array}{c}\text { Star } \\
\text { Names }\end{array}$ & $\begin{array}{l}\text { R.A.(J2000) } \\
\text { DEC.(J2000) }\end{array}$ & $\begin{array}{c}\pi_{r e l} \\
(\mathrm{mas})\end{array}$ & $\begin{array}{c}\Delta \pi \\
(\mathrm{mas})\end{array}$ & $\begin{array}{l}\pi_{a b s} \\
(\mathrm{mas})\end{array}$ & $\begin{array}{c}\mu_{r e l} \\
\left(\operatorname{mas~yr}^{-1}\right)\end{array}$ & $\begin{array}{c}\text { PA } \\
(\operatorname{deg})\end{array}$ & $\mathrm{V}$ & $\begin{array}{l}\mathrm{B}-\mathrm{V} \\
\mathrm{V}-\mathrm{I}\end{array}$ & $\mathrm{K}^{\mathrm{b}}$ & $\begin{array}{l}\mathrm{J}-\mathrm{H} \\
\mathrm{H}-\mathrm{K}\end{array}$ \\
\hline LP765-18 & 002600.5 & 6.88 & 1.08 & 7.96 & 613.2 & 180.92 & 15.07 & 1.69 & 11.64 & 0.58 \\
\hline LHS1075 & -191852 & \pm 0.80 & \pm 0.24 & \pm 0.84 & \pm 0.6 & \pm 0.03 & & 1.61 & & 0.32 \\
\hline LP328-57 & 155237.4 & 3.25 & 1.29 & 4.54 & 278.4 & 228.00 & 16.30 & 1.67 & 12.82 & 0.61 \\
\hline CLS96 & +292800 & \pm 0.56 & \pm 0.35 & \pm 0.66 & \pm 0.2 & \pm 0.04 & & 1.57 & & 0.32 \\
\hline $\mathrm{G} 77-61^{\mathrm{c}}$ & 033238.0 & 15.0 & 1.9 & 16.9 & 772.5 & 165.80 & 13.89 & 1.75 & 10.49 & 0.64 \\
\hline LHS1555 & +015800 & \pm 2.2 & \pm 0.4 & \pm 2.2 & \pm 0.6 & \pm 0.10 & & 1.56 & & 0.36 \\
\hline
\end{tabular}

a Positions are given for epoch and equinox 2000.0 based on Deutsch 1994.

bJHK photometry on the CTIO system taken from Bothun et al. 1991 and Dearborn et al. 1986.

${ }^{\mathrm{c}}$ For G77-61, the astrometry and BVI photometry are from Dahn et al. 1977 and Dearborn et al. 1986.

Table 2. Derived Results.

\begin{tabular}{crccrcrc}
\hline \hline Star & \multicolumn{1}{c}{$\mathrm{M}_{\mathrm{V}}$} & $\mathrm{M}_{\mathrm{K}}$ & $\begin{array}{c}\mathrm{V}_{\text {tan }} \\
\left(\mathrm{km} \mathrm{s}^{-1}\right)\end{array}$ & $\begin{array}{c}\mathrm{V}_{\mathrm{r}} \\
\left(\mathrm{km} \mathrm{s}^{-1}\right)\end{array}$ & $\begin{array}{c}\mathrm{V} \\
\left(\mathrm{km} \mathrm{s}^{-1}\right)\end{array}$ \\
\hline LP765-18 & $9.58 \pm 0.23$ & $6.14 \pm 0.23$ & $365 \pm 39$ & $56 \pm 19$ & 205 & -282 & -98 \\
LP328-57 & $9.59 \pm 0.32$ & $6.11 \pm 0.32$ & $291 \pm 43$ & $164 \pm 10$ & 148 & -164 & 252 \\
G77-61 & $10.03 \pm 0.28$ & $6.63 \pm 0.28$ & $217 \pm 43$ & $-34 \pm 1$ & 106 & -176 & -40 \\
\hline
\end{tabular}




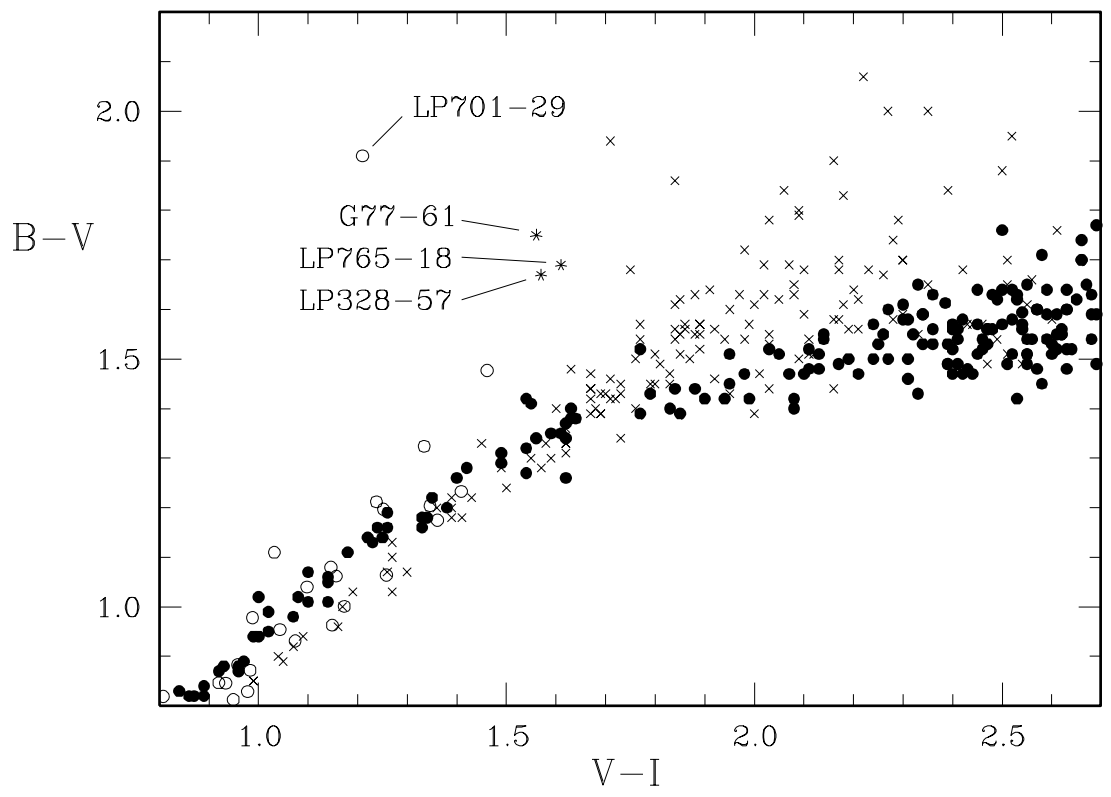

Fig. 1.- The unusual colors of dC stars (asterisks), compared with disk dwarfs (filled circles), spheroid subdwarfs (x's), and white dwarfs (open circles). 


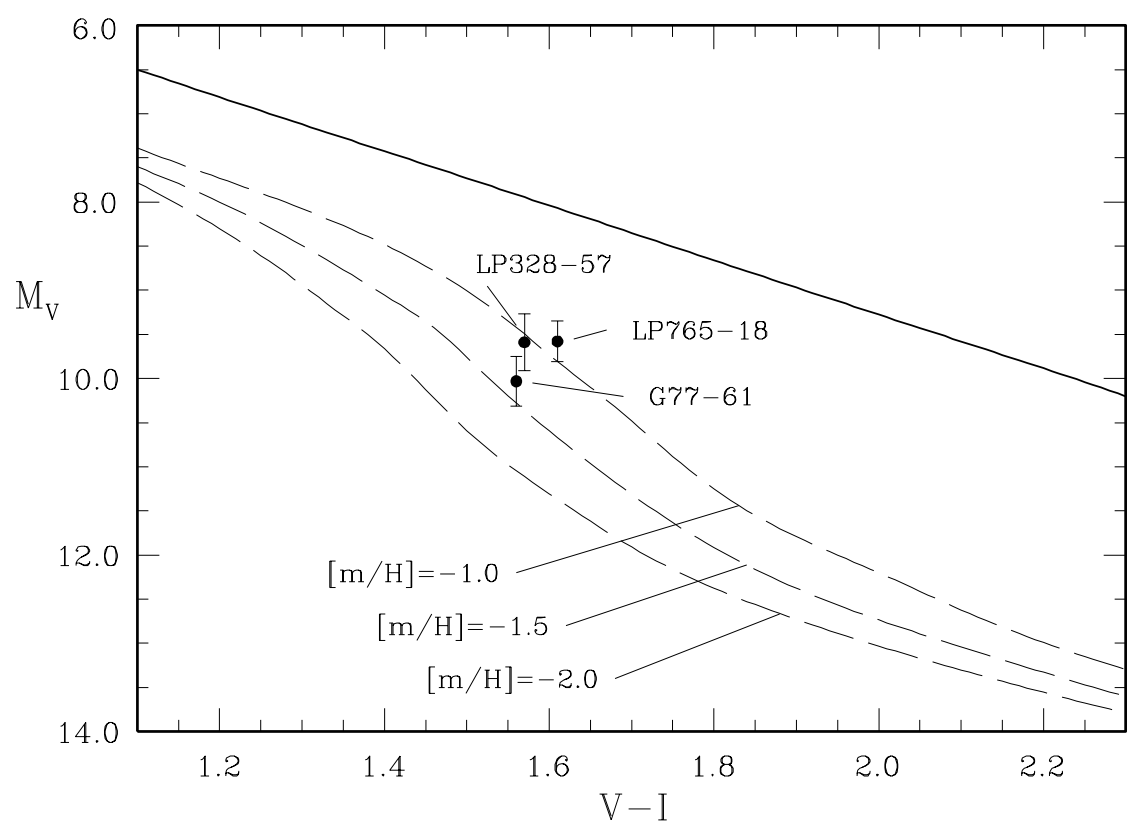

Fig. 2.- The absolute $\mathrm{V}$ magnitude of the three dC stars with measured parallaxes. 


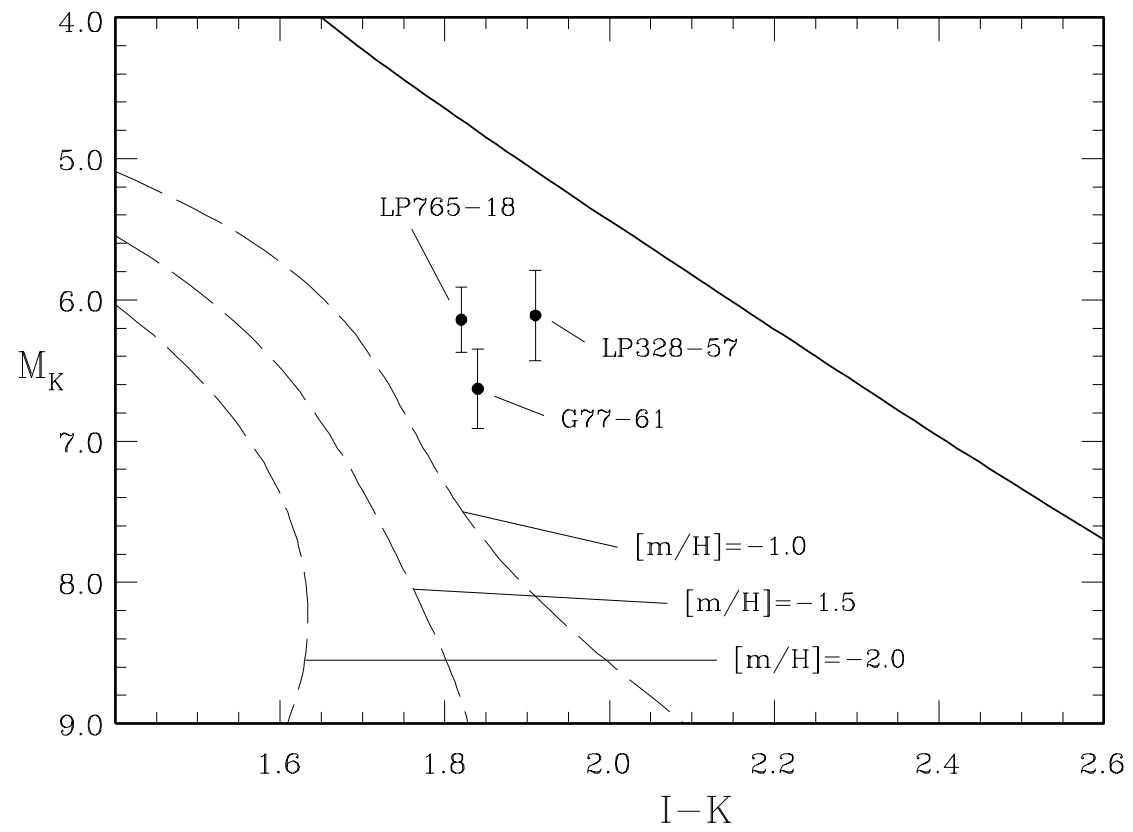

Fig. 3.- The absolute K magnitude of the three dC stars. 


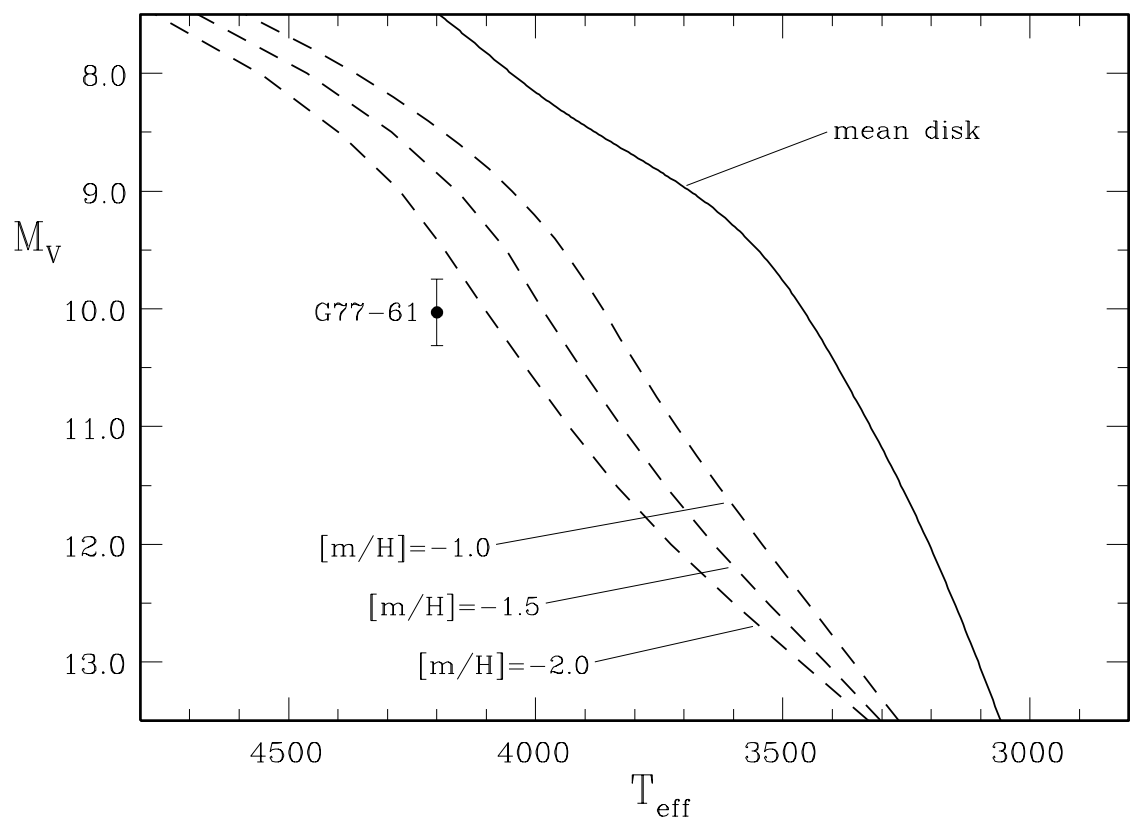

Fig. 4. - The absolute $\mathrm{V}$ magnitude against effective temperature of the one dC star with a measured temperature. 